\title{
High Gain, Low Cost, Low Profile Transmitarray Antenna
}

\author{
Mário S. V. Arigony ${ }^{1,2}$, Candice Müller ${ }^{1,3}$, Fernando C. C. de Castro ${ }^{1,4}$, Natanael R. Gomes ${ }^{1,5}$ e Samuel T. \\ Valduga ${ }^{1,6}$
}

\begin{abstract}
This paper presents a double layer double ring unit cell for circular polarization transmitarrays. The proposed unit cell is evaluated with Rogers 4003 and FR4 substrates. Both models are applied to the design of a $13 \times 13$ elements transmitarray with a log spiral feeder at $10 \mathrm{GHz}$. Results show that the transmitarray achieves a directivity of $20.7 \mathrm{dBi}$ and $20.0 \mathrm{dBi}$ at the boresight, with Rogers 4003 and FR4 substrates, respectively, which is nearly $12 \mathrm{~dB}$ above the log spiral gain. The results of the proposed transmitarray are compared with a horn antenna and a toroidal plasma lens. When compared with solutions that present equivalent boresight directivity, the proposed transmitarray achieves significantly reduced profile, showing to be a high gain low cost, low profile and easy to prototype solution.
\end{abstract}

Keywords-Transmitarray, antenna, metamaterials.

\section{INTRODUCTION}

High gain, high frequency and circular polarization are antenna characteristics generally obtained by complex and costly waveguide based solutions. A planar element combined with dielectric lenses may represent a cheaper solution for some applications [1]. In this context, a transmitarray is a promising alternative to high gain antennas, such as dielectric lenses, parabolic reflectors and phased arrays. A transmitarray is the combination of a feeding antenna and an array of unit cells, as shown in Fig. 1. The unit cell is composed of conventional materials, such as metals and dielectrics, arranged in a specific geometric pattern. The array of unit cells forms the electromagnetic metamaterial [2]. The ability to control the electromagnetic wave properties, with different metamaterial structures, had significant impact on the development of advanced microwave devices in the last years [3-8].

The transmitarray is basically a phase-shifting surface capable of focusing the electromagnetic (EM) wave by means of constructive interference, producing a high gain beam [9-11]. Each unit cell compensates the phase delay of the incident EM wave, according to its spatial position in the array, in such a way that the EM waves transmitted by each unit cell element are in phase, forming a coherent beam. Thus, ideally, the unit cells should be able to shift the phase of the transmitted EM wave in the full range of $2 \pi$ radians.

The array of unit cells is placed in front of the feeding antenna. The phase of the EM wave which impinges each unit cell of the array is shifted by a specific value [11]. The unit cells are generally composed by resonant elements, typically inspired by metamaterials and frequency selective surfaces, and it works similarly to dielectric lenses [9]. Low profile, low mechanical complexity, compactness, no feeding network and simplicity in

${ }^{1}$ Department of Electronics and Computer, Federal University of Santa Maria, Santa Maria-RS; e-mails: ${ }^{2}$ mariovarigony@gmail.com; ${ }^{3}$ candice.muller@ufsm.br ${ }^{4}$ fernando.castro@ufsm.br; ${ }^{5}$ natanael.rgomes@gmail.com; ${ }^{6}$ samucatv@gmail.com. This study was partially financed by Conselho Nacional de Desenvolvimento Científico e Tecnológico (CNPq, Brazil). prototyping are some advantages of transmitarrays when compared to dielectric lenses, parabolic reflectors and phased arrays.

In this context, this paper presents a double layer double ring unit cell for circular polarization. The proposed unit cell is evaluated with low loss Rogers 4003 substrate and low cost FR4 substrate. Both models are then applied to a $13 \times 13$ transmitarray with a log spiral feeder at $10 \mathrm{GHz}$. Results show that the transmitarray achieves a gain of $20.7 \mathrm{dBi}$ and $20.0 \mathrm{dBi}$ at the boresight, with Rogers 4003 and FR4 substrates, respectively, which is nearly $12 \mathrm{~dB}$ above the log spiral gain. The results of the proposed transmitarray are compared with a horn antenna and a toroidal plasma lens. The comparison shows that, with equivalent gain, the transmitarray presents significantly reduced profile, showing to be a low cost and easy to prototype solution.

The rest of this paper is organized as follows: Section II shows the proposed unit cell. The developed transmitarray antenna is presented in Section III. Section IV compares the results and Section V brings the conclusions.

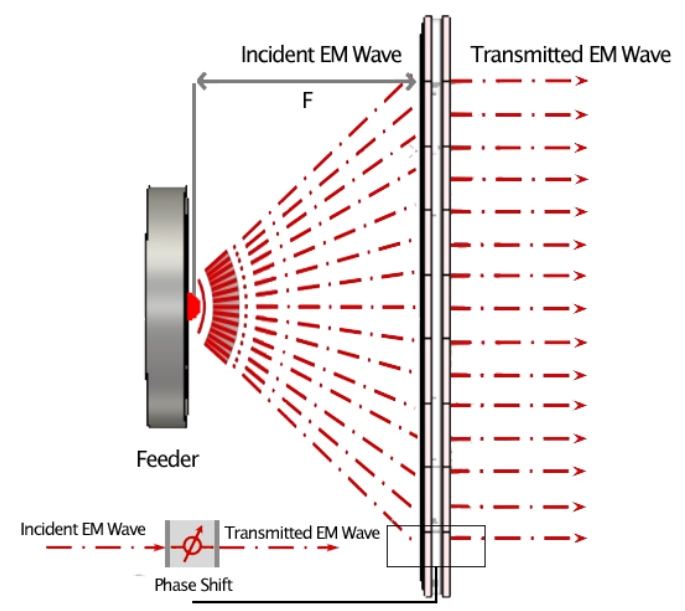

Fig. 1. Transmitarray working principle.

\section{PROPOSED UNIT CELL}

The proposed unit cell is a double layer circularly polarized cell that applies the element rotation method for the phase shift. Each layer is composed by two concentric rings printed on a dielectric substrate. The inner ring is a split-ring and the rotation of the ring gap on the substrate plane controls the phase shift of the transmitted EM wave. The layers are separated by air and the printed rings are placed face to the face, to the internal side of the unit cell, as shown on Fig. 2. The unit cell has been modeled and simulated using finite-difference time-domain (FDTD) simulation algorithm. 


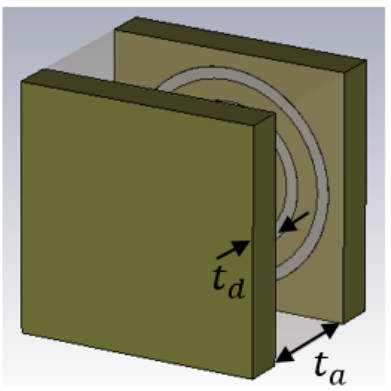

(a)

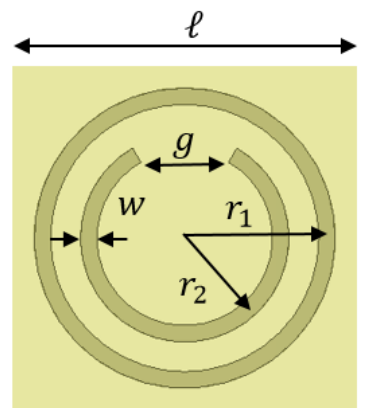

(b)
Fig. 2. (a) Unit cell perspective view; (b) Detailed view of the double ring faces.

The unit cell behavior has been evaluated with Rogers 4003, low loss dielectric (loss tangent $\tan \delta=0.0027$ ) and FR4, higher loss $(\tan \delta=0.025)$, low cost and easy to buy dielectric.

The dimensions of the proposed structure have been optimized at $10 \mathrm{GHz}$ by using genetic algorithm, aiming to reduce the insertion loss. The resulting dimensions are presented in Table I. $\ell$ is the cell width, $r_{1}$ and $r_{2}$ are the outer and inner ring radius, respectively, $w$ is the rings width, $g$ is the inner ring gap, $t_{d}$ is the dieletric thickness and $t_{a}$ is the air layer thickness. The optimized cell width $\ell$ resulted in approximately $0.42 \lambda$ at $10 \mathrm{GHz}$.

Fig. 3 presents the transmission coefficient $\tau$ versus frequency. Each curve refers to an inner ring rotation angle $\alpha$. Fig. 4 presents the phase shift of the transmitted electromagnetic wave versus the rotation of inner split-ring on the substrate plane, given by angle $\alpha$, obtained with Rogers 4003 and FR4 dielectrics.

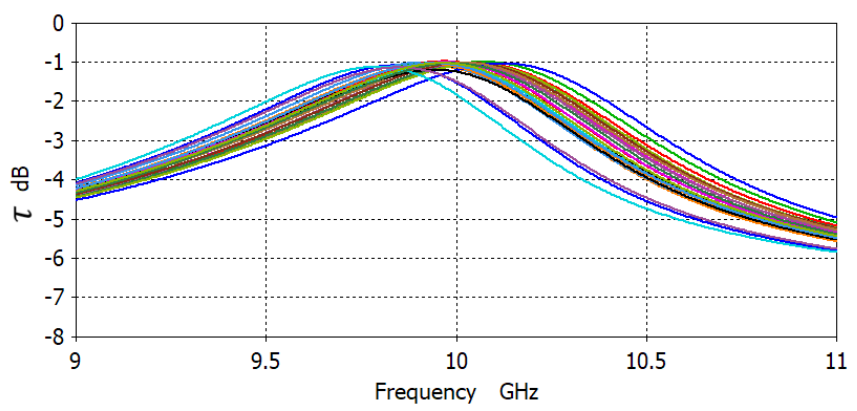

(a)

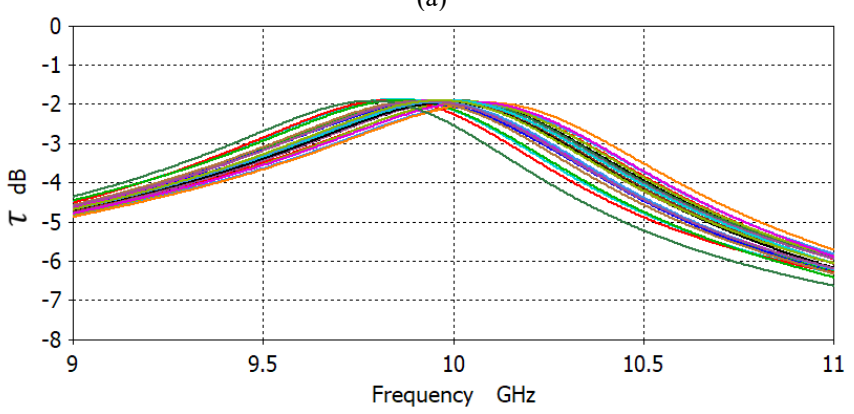

(b)

Fig. 3. Transmission coefficient $\tau$ using (a) Rogers 4003; (b) FR4. Each curve refers to an inner ring rotation angle $\alpha$.
TABLE I. Unit Cell Parameters

\begin{tabular}{|l|c|c|c|c|c|c|c|c|c|}
\hline & $\ell$ & & $r_{1}$ & $r_{2}$ & $w$ & $g$ & $t_{d}$ & $t_{a}$ & $\epsilon_{r}$ \\
\hline $\begin{array}{l}\text { Rogers } \\
4003\end{array}$ & 12.5 & & 5.7 & 3.9 & 0.45 & 3.74 & 1.5 & 2.5 & 3.55 \\
\hline FR4 & 12.5 & & 5.7 & 3.9 & 0.54 & 4.07 & 1.5 & 2.0 & 4.3 \\
\hline
\end{tabular}

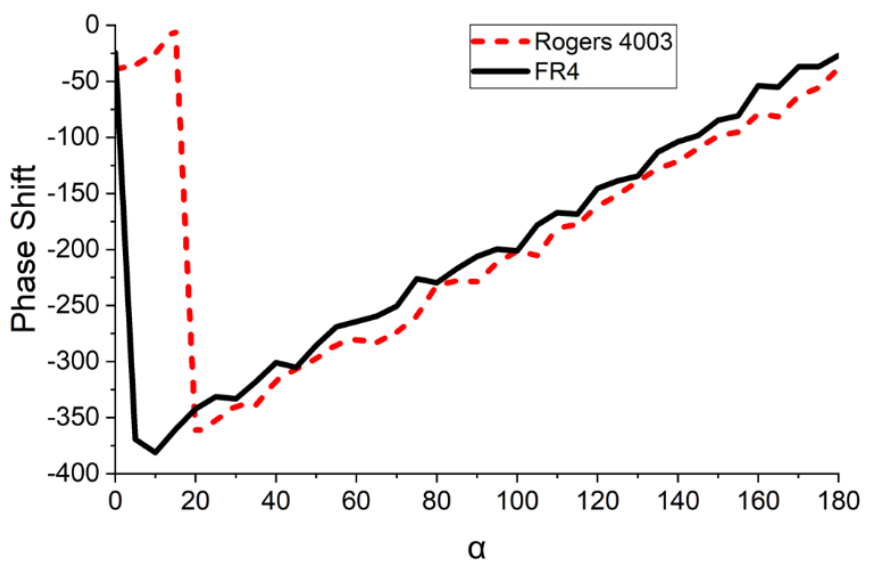

Fig. 4. Phase shift of the transmitted electromagnetic wave versus the rotation of inner split-ring on the substrate plane.

As expected, the results obtained with Rogers 4003 are slightly superior than the results obtained with FR4. $\tau$ presents maximum values of $-0.99 \mathrm{~dB}$ and $-1.90 \mathrm{~dB}$ for Rogers 4003 and FR4, respectively, which means that FR4 presents approximately $1 \mathrm{~dB}$ increase in insertion loss. Observe that $\tau$ is minimally affected by the inner split-ring rotation angle $\alpha$. The unit cell bandwidth is reduced when applying FR4: Rogers 4003 presents $9.0 \%$ bandwidth while for FR4 it is reduced to $6.0 \%$. Both structures present quite linear phase shift of the transmitted EM wave. Note that it is possible to reach the phase shift range from $0^{\circ}$ to $360^{\circ}$ with a gentle phase slope, which allows minimizing manufacturing errors.

\section{TRANSMITARRAY ANTENNA}

The proposed unit cell presented in Section II has been applied to the design of a $13 \times 13$ elements transmitarray. The inner split-ring rotation $\varphi_{i}$ of the $i^{\text {th }}$ unit cell, which controls the phase shift of the transmitted EM wave, is the phase difference between the phase of the incident EM wave of the $i^{\text {th }}$ unit cell $\theta_{i}$ and the incident EM wave of central unit cell $\theta_{c}$ of the array. $\varphi_{i}$ and $\theta_{i}$ are given by

$$
\begin{gathered}
\varphi_{i}=\theta_{i}-\theta_{c} \\
\theta_{i}=R_{i} \beta-r_{0} \beta
\end{gathered}
$$

where, $R_{i}$ is the distance from the feeder to the $i^{\text {th }}$ unit cell element, given by $R_{i}=\sqrt{r_{i}^{2}+r_{0}^{2}}$, where $r_{i}$ is the distance between the central element and the $i^{t h}$ element, which is given by $r_{i}=\sqrt{x^{2}+y^{2}}$, where $x$ and $y$ are the coordinates of the $i^{t h}$ element, considering that the central element is located at coordinates $x=0, y=0$. The distance between the feeder and the central element of the transmitarray is represented by $r_{0} . \beta$ represents to the propagation constant in the free space, given by 
$\beta=2 \pi / \lambda$, where $\lambda=c / f$ is the wavelength. Fig. 5 shows the required phase shift of each array element.

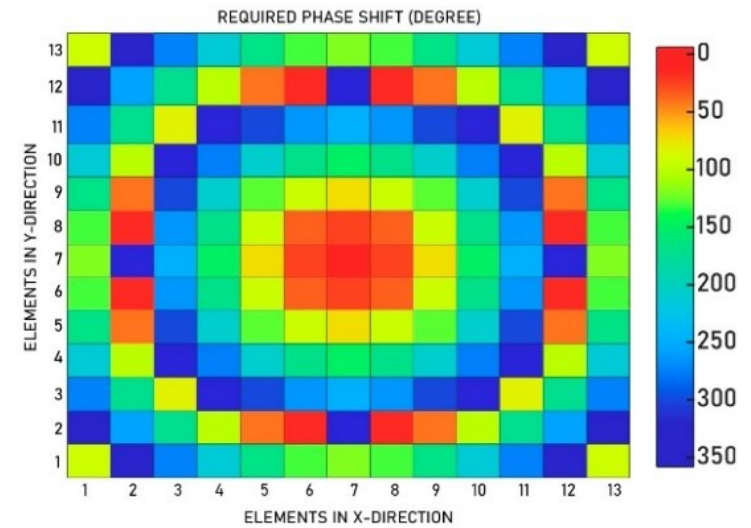

Fig. 5. Required phase shift of each unit cell of the Transmitarray.

The feeder is a $\log$ spiral antenna, placed at a distance $F=$ $50 \mathrm{~mm}$ from the array plane. Fig. 6 presents the transmitarray model. Note the inner split-ring rotation of each unit cell, which controls the phase shift of the transmitted EM wave.

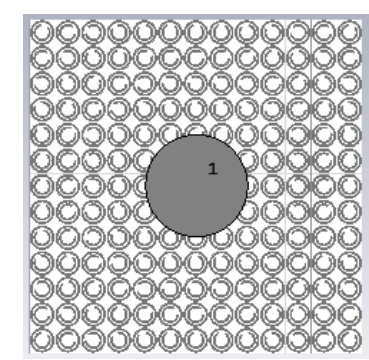

(a)

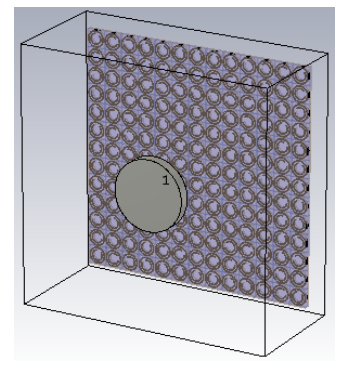

(b)
Fig. 6. (a) Transmitarray front view; (b) Transmitarray perspective view.

Fig. 7 compares the radiation pattern of the log spiral antenna and the radiation pattern of the transmitarray with Rogers 4003 substrate, both operating at $10 \mathrm{GHz}$. Note that the transmitarray presents $20.7 \mathrm{dBi}$ boresight directivity, $-11.3 \mathrm{~dB}$ side lobe level (main lobe gain in $\mathrm{dB}$ minus maximum secondary lobe gain in $\mathrm{dB})$ and $12.2^{\circ}$ angular width $(3 \mathrm{~dB})$, while for the log spiral antenna the boresight directivity is only $8.41 \mathrm{dBi}$, the side lobe level is $-16.2 \mathrm{~dB}$ and the angular width is $73.7^{\circ}$.

Fig. 8 compares the radiation pattern of the log spiral antenna and the radiation pattern of the transmitarray with FR4 substrate, both operating at $10 \mathrm{GHz}$. The transmitarray presents $20.0 \mathrm{dBi}$ boresight gain, $-8.3 \mathrm{~dB}$ side lobe level and $12.1^{\circ}$ angular width $(3 \mathrm{~dB})$. Thus, the transmitarray efficiently adjusted the phase of the incident waves from log spiral source so that the re-irradiated waves interfere constructively, increasing the beam gain in approximately $12.3 \mathrm{~dB}$ and $11.6 \mathrm{~dB}$ with Rogers 4003 and FR4 substrates, respectively.

Fig. 9 presents the transmitarray antenna gain over frequency (losses included). Fig. 9 (a) presents the results for Rogers 4003 substrate and Fig. 9 (b) for FR4 substrate. The evaluated transmitarrays present $14.5 \%$ bandwidth (Rogers 4003 ) and $17 \%$ bandwidth (FR4).

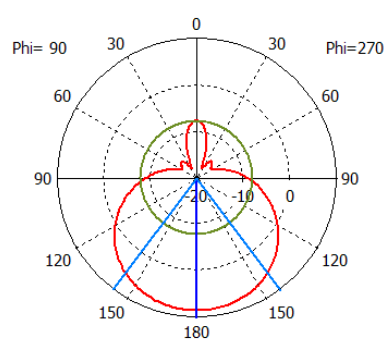

(a)

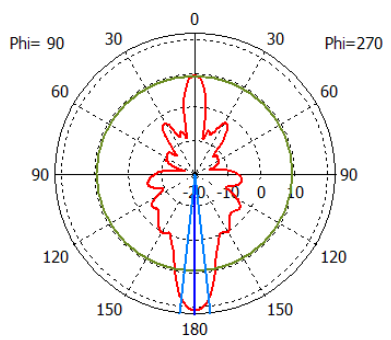

(b)
Fig. 7. Antennas directivity at $10 \mathrm{GHz}$ (a) Log spiral antenna: boresight directivity $=8.41 \mathrm{dBi}$; angular width $(3 \mathrm{~dB})=73.7^{\circ}$; side lobe level $=-16.2$ $\mathrm{dB}$; (b) Rogers 4003 substrate transmitarray: boresight directivity $=20.7 \mathrm{dBi}$; angular width $(3 \mathrm{~dB})=12.2^{\circ}$; side lobe level $=-11.3 \mathrm{~dB}$

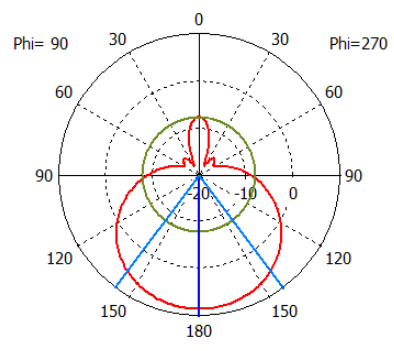

(a)

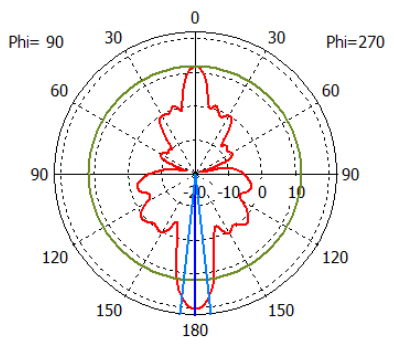

(b)
Fig. 8. Antennas directivity at $10 \mathrm{GHz}$ (a) Log spiral antenna: boresight directivity $=8.41 \mathrm{dBi}$; angular width $(3 \mathrm{~dB})=73.7^{\circ}$; side lobe level $=-16.2$ $\mathrm{dB}$; (b) FR4 substrate transmitarray: boresight directivity $=20.0 \mathrm{dBi}$; angular width $(3 \mathrm{~dB})=12.1^{\circ}$; side lobe level $=-8.3 \mathrm{~dB}$.

The transmitarray performance with Rogers 4003 and FR4 resulted similar in terms of boresight directivity and angular width. Rogers 4003 substrate achieved approximately $3 \mathrm{~dB}$ reduced side lobe and up to $2 \mathrm{~dB}$ higher antenna gain compared to FR4 substrate. On the other hand, FR4 substrate reaches larger bandwidth than Rogers 4003 substrate.

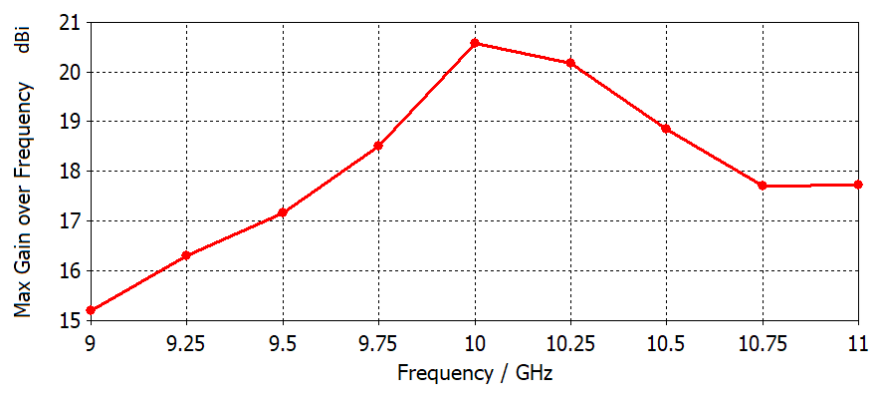

(a)

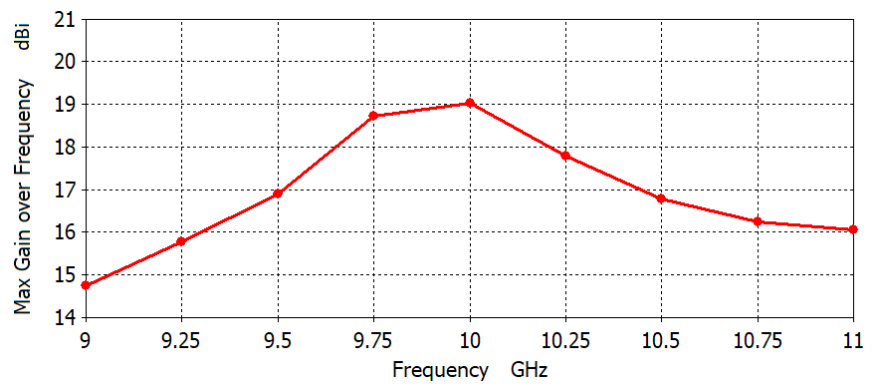

(b)

Fig. 9. Transmitarray maximum gain over frequency: (a) Rogers 4003 substrate; (b) FR4 substrate. 


\section{RESULTS COMPARISON}

A widely adopted solution for circular polarization and high gain applications is the horn antenna [1]. The toroidal plasma lens (TPL) is an alternative solution that presents low profile and low radar cross section [13]. Thus, the results of the proposed transmitarray are compared with the horn antenna and TPL.

The transmitarray profile is evaluated considering the antenna volume, i.e., the volume of a box into which the whole antenna fits exactly. The transmitarray dimensions are $16.25 \mathrm{~cm} \times 16.25 \mathrm{~cm} \times 6.3 \mathrm{~cm}$, resulting in approximately $1.66 \times 10^{3} \mathrm{~cm}^{3}$.

As for the overall physical size, the transmitarray presents significantly smaller length and volume when compared to a conical horn of same gain. A conical horn optimized for $20 \mathrm{dBi}$ at $10 \mathrm{GHz}$ has a diameter of $133 \mathrm{~mm}$ and a length of $185 \mathrm{~mm}$ [12]. Actually, in practice, the horn will have a larger length when considering the coaxial-to-waveguide adapter, polarizer, and the waveguide connections. Thus, the conical horn has approximately three times the length of the transmitarray, and its volume is approximately 2.5 times larger than the transmitarray volume. Therefore, the proposed transmitarray presents a significantly smaller profile, keeping the same gain of a larger profile conical horn antenna.

Comparing the $19.9 \mathrm{dBi}$ TPL [13] profile with the $20 \mathrm{dBi}$ transmitarray profile, the TPL overall physical size is larger than the transmitarray size. TPL is approximately $15 \%$ wider than transmitarray, $60 \%$ longer and its volume is more than two times the transmitarray volume.

\section{CONCLUSION}

This paper presents a double layer double ring unit cell for circular polarization that applies the element rotation method to control the phase shift of the transmitted wave. The unit cell has been modeled and optimized for $10 \mathrm{GHz}$ operating frequency. The model has been evaluated with low cost FR4 substrate and with low loss Rogers 4003 substrate. Results show that Rogers substrate achieves nearly $1 \mathrm{~dB}$ insertion loss reduction and larger bandwidth when compared to FR4.

Based on the proposed unit cell model, a $13 \times 13$ transmitarray has been designed, modeled and simulated. The feeder element is a log spiral antenna. The metamaterial transmitarray achieves $20.7 \mathrm{dBi}$ and $20.0 \mathrm{dBi}$ boresight directivity with Rogers 4003 and FR4 substrates, respectively, which is approximately $12 \mathrm{~dB}$ above the log spiral antenna directivity. The results obtained from Rogers 4003 and FR4 are similar in terms of boresight directivity and angular width. The FR4 transmitarray achieves larger bandwidth than Rogers 4003 transmitarray. Rogers 4003 substrate achieved approximately 3 $\mathrm{dB}$ reduced side lobe and up to $2 \mathrm{~dB}$ higher antenna gain compared to FR4 substrate. The results show that FR4 is an attractive solution due to its low cost and good performance.

The transmitarray results are compared to horn antenna and a toroidal plasma lens. Achieving similar boresight directivity, the transmitarray has significantly reduced profile when compared to the horn and TPL. Thus, the proposed transmitarray shown to be a high gain, compact and low cost and solution with the advantage of having a simple manufacturing process.

\section{ACKNOLEDGES}

The authors would like to thanks the Conselho Nacional de Desenvolvimento Científico e Tecnológico (CNPq, Brazil) for the partial financial support of this study.

\section{REFERÊNCIAS}

[1] R. C. Johnson, “Antenna engineering handbook”. 3rd. ed. New York: McGraw-Hill, 1993.

[2] I.A. BURIAK, O.O. RYBALKO, V.O. ZHURBA. "Metamaterials: Theory, Classification and Application Strategies". J. NANOELECTRON. PHYS. 8, 04088, 2016.

[3] N. Engheta, R.W. Ziolkowski, Metamaterials: Physics and Engineering Explorations. New York: IEEE Wiley, 2006.

[4] Metamaterials Handbook: Vol. I. Phenomena and Theory of Metamaterials. Boca Raton: Taylor \& Francis, 2009.

[5] G.V. Eleftheriades, K.G. Balmain, Negative-refraction metamaterials: Fundamental Principles and Applications. New York: IEEE-Wiley, 2005.

[6] T. Itoh and C. Caloz. "Electromagnetic metamaterials: transmission line theory and microwave applications". New Jersey, USA: John Wiley $\backslash \&$ Sons, Inc., 2006.

[7] I.B Vendik, O.G. Vendik, Tech. Phys. 58(1), 1, 2013.

[8] V.I. Slyusar, Electronics: Science, Technology, Business, 7, 70, 2009.

[9] J. R. Reis, "Review Paper on Transmitarray Antennas". IEEE Access, Julho 2019.

[10] B. Rahmati and H. R. Hassani, "High-efficient wideband slot transmitarray antenna," IEEE Transactions on Antennas and Propagation, vol. 63, no. 11, pp.5149-5155, 2015.

[11] 2 A. H. Abdelrahman, F. Yang, A.Z. Elsherbeni, and P. Nayeri, "Analysis and Design of Transmitarray Antennas", Morgan Claypool, Synthesis Lectures on Antennas, January 2017, Vol. 6, No. 1, 1st ed., San Rafael, CA, USA, pp. 7-12, 39-47, 2017.

[12] A. A. Nafati, C. A. Balanis, C. R. Birtcher, "Conical horn: gain and amplitude patterns.", IEEE Transactions on Antennas and Propagation, Hong Kong, v. 61, n. 7, 2013.

[13] M. O. Arend, F. C. C. De Castro, C. Muller, M. C. F. Castro, "Toroidal Plasma Lens Antenna", Antennas and Wireless Propagation Letters, v. 1, p. 1-4, 2016. 